\title{
Women with breast cancer decided whether to use complementary/alternative medicine through a process of discovery and investigation
}

Boon H, Brown JB, Gavin A, et al. Breast cancer survivors' perceptions of complementary/alternative medicine (CAM): making the decision to use or not to use. Qual Health Res 1999 Sep;9:639-53.

QUESTION: What are the experiences of women with breast cancer as they decide whether to use complementary/alternative medicine (CAM) (use of natural healthcare products, such as herbs and nutritional supplements, and the process of seeking advice from non-conventional healthcare professionals, such as homeopaths and acupuncturists)?

\section{Design}

Focus groups.

\section{Setting}

London and Toronto, Ontario, Canada.

\section{Participants}

36 women (age range 41-73 y) diagnosed with breast cancer at some time in their lives were recruited through notices to regional support groups, cancer centres, health food stores, and pharmacies. Most were white, and middle to upper middle class. They had been diagnosed an average of 5 years before the study (range 8 mo to 15 y). 11 women identified themselves as non-users of CAM and 25 as users.

\section{Methods}

3 focus groups were held in each site. During the 2 hour audiotaped discussions, women were asked about their perceptions and experiences regarding their use or non-use of CAM. Data collection continued until saturation. Verbatim transcripts were content analysed independently by 4 of the researchers, analyses were combined, and key emerging themes were confirmed by 2 breast cancer survivors.

\section{Main findings}

Key themes included the discovery and investigation of CAM, the decision making process, and barriers to using CAM. After diagnosis, women began a process of discovering CAM through word of mouth, the media, conventional and CAM practitioners, and personal reading. After they discovered CAM, women investigated the treatment by reading the literature and talking with breast cancer survivors and practitioners. The decision to use or not use CAM was a highly individualised process. Women recognised their personal responsibility for decision making and noted the need for guidance. Some physicians were supportive, whereas others were sceptical or actively discouraged the use of CAM. Women reported that friends and family served as sounding boards and provided emotional and financial support. Reasons for deciding not to use CAM included inadequate information, a perception that the treatments were not effective, and fear that they could be harmful. Reasons for deciding to use CAM were: 1) improved chance of survival; 2) reaction to bad experiences with conventional medicine (eg, treatment failure or adverse effects); 3) prevention of further illness (based on a belief that CAM could boost the immune system); and 4) belief that they had "nothing to lose" because CAM was not harmful. Some women who decided to use CAM reported encountering several barriers: cost of treatment because CAM was usually not covered by government healthcare plans; lack of access because treatments were not directly accessible or required a physician's order; and the large amount of time required for such things as multiple visits to practitioners, adherence to treatment schedules, and preparation of special diets.

\section{Conclusions}

Women with breast cancer went through a process of discovery and investigation when deciding whether to use complementary/alternative medicine (CAM). Those who decided to use CAM saw potential benefits and no harmful effects; those who decided not to use CAM had explicitly considered the risks.

\section{COMMENTARY}

This study by Boon $e t$ al offers insight into the process women used to make decisions about CAM in a time of increased publicity and attention to CAM treatments. Data were generated in focus groups with a fairly homogeneous group of white, middle to upper middle class women. Other demographic information, such as education level or marital status, was not provided. These findings may not apply to women who differ from those included here. The careful process of data collection and analysis, however, strengthens the results.

One important aspect of this study is that all of the participants at least considered $\mathrm{CAM}$ as an adjunct to their treatment. The authors developed a model that identifies the complexity of the individual decision making process. This model may be helpful for those working with breast cancer survivors to understand and support their process of discovery.

It cannot be assumed that all women with a diagnosis of cancer will consider CAM. This study does highlight, however, the need for healthcare professionals to be knowledgeable about CAM practices in order to provide comprehensive and holistic care. Participants who elected not to pursue CAM were presented as mostly having "risk awareness", whereas those who used CAM were identified in part as feeling that they "had nothing to lose". The treatments considered by the participants were mostly those that involved ingested substances, such as herbs and other supplements, which indeed might have potential risks. This suggests that it is important to assist clients in all aspects of their decision making. CAM treatments that are described as having no inherent risk (ie, energy based therapies) may not fit this model of risk awareness.

This study shows the importance of open communication and respect for clients as they make treatment choices. It also provides support for the increased use of CAM as reported by Eisenberg $\mathrm{et} \mathrm{al},{ }^{1}$ and gives additional information about the decision making process that is not found in quantitative studies such as Burstein $e t a l l^{2}$ which reported increased psychosocial distress and poorer quality of life among breast cancer patients who used CAM treatments.

Diane Wardell, RNC, PhD Associate Professor, School of Nursing University of Texas at Houston Houston, Texas, USA

1 Eisenberg DM, Davis RB, Ettner SL, et al. Trends in alternative medicine use in the United States, 1990-1997: results of a follow-up national survey. JAMA 1998;280:1569-75.

2 Burstein HJ, Gelber S, Guadagnoli E, et al. Use of alternative medicine by women with early-stage breast cancer. $N$ EnglJ Med 1999;340:1733-9. 